\title{
Integrated genomic and methylation profile analysis to identify candidate tumor marker genes in patients with colorectal cancer
}

\author{
GUOJUN HUANG $^{1 *}$, WANG $\mathrm{CHENG}^{2 *}$ and FU XI ${ }^{1}$ \\ Departments of ${ }^{1}$ Oncology and ${ }^{2}$ General Surgery, Pidu District People's Hospital, Chengdu, Sichuan 611730, P.R. China
}

Received January 6, 2019; Accepted July 11, 2019

DOI: $10.3892 / \mathrm{ol} .2019 .10799$

\begin{abstract}
Aberrant genomic expression and methylation serve important roles in cancer development. Integrated analysis of genetic and methylation profiles may identify potential tumor marker genes for colorectal cancer (CRC) prediction. In the current study, DNA methylation and mRNA expression profiles associated with CRC were downloaded from The Cancer Genome Atlas database. Differentially expressed mRNAs and methylated genes between tumor samples and adjacent healthy tissues were identified. Candidate tumor marker genes and prognostic clinical factors were screened according to univariable and multivariable Cox regression analysis. A total of 218 DEGs with aberrant methylation levels were screened from tumor samples. A risk prediction model was constructed based on identified genes and clinical factors. Randomization tests were used to evaluate the performance of the prediction model, including area under the curve (AUC) calculation and cross-validation. Cox regression analysis revealed that eight genes and six prognostic clinical factors were significantly associated with survival outcomes. Functional and pathway enrichment analysis revealed that the eight genes were mainly involved in 'cell adhesion', 'fatty acid metabolism' and 'cytokine receptor interaction' pathways. After combining six clinical factors with eight genes, the accuracy of risk prediction model has been increased intensively. The P-values representing the association between risk grouping and prognosis decreased from 0.009 to 0.001 and the AUC increased from 0.992 to 0.999 , indicating that the comprehensive risk prediction model exhibited a good performance for disease prognosis prediction. The current study integrated genomic and methylation profiles and identified eight tumor marker
\end{abstract}

Correspondence to: $\mathrm{Dr} \mathrm{Fu} \mathrm{Xi}$, Department of Oncology, Pidu District People's Hospital, 156 East Pitong Street, Chengdu, Sichuan 611730, P.R. China

E-mail: fuxijujiafei@126.com

*Contributed equally

Key words: colorectal cancer, DNA methylation, prognosis, risk prediction model genes in CRC. These candidate genes may improve the prediction accuracy of CRC prognosis.

\section{Introduction}

Colorectal cancer (CRC) is one of the most common malignant tumors and it has exhibited an increasing morbidity rate in the past decades. In 2017, 135,000 individuals were diagnosed with CRC in the USA, and the mean 5-year survival rate was $<10 \%$ once metastasis occurred $(1,2)$. An accumulation of genetic and epigenetic changes, including somatic mutations in the B-Raf proto-oncogene serine/threonine kinase, KRAS proto-oncogene GTPase and tumor protein 53 genes, contribute to the tumorigenesis of CRC. These mutations are observed in the majority of patients with CRC (3). Additionally, several signaling pathways, including Wnt, Notch, mitogen-activated protein kinase, transforming growth factor- $\beta$ and phosphatidylinositol 3-kinase/protein kinase b pathways, are involved in the oncogenic transformation of CRC (4-6). Furthermore, the pathogenesis of a subset of CRC involves mechanisms such as aberrant DNA methylation or $\mathrm{CpG}$ island methylator phenotype (CIMP) in promoter methylation (7).

Previous studies have reported that DNA methylation functions as a key regulator of gene expression and contributes to CRC development $(8,9)$. At the genome level, CRC is characterized by absolute hypomethylation compared with adjacent healthy tissues, such as hypomethylation level in repetitive elements, including long interspersed nuclear element-1 and the Alu element (10). Furthermore, a subset of patients with CRC exhibit gene-specific promoter methylation termed CIMP (11). While a number of methylated genes are established as tumor suppressors in CRC, the potential roles of several methylated genes in tumorigenesis remain unclear. For example, McInnes et al (12) analyzed a subset of DNA methylation profiles and identified a cohort of hypermethylated genes in high level CIMP CRC tissues. Xue et al (13) used genome-wide methylation analysis to screen several novel methylated markers with prognostic value in colon cancer and revealed that five genes coincided with seven prognostic differentially expressed regions. However, the use of potential DNA methylation genes as predictive markers in CRC detection and prognosis prediction requires further investigation.

The current study analyzed mRNA expression and methylation profiles to screen differentially expressed mRNAs and methylated genes between CRC tumors and adjacent 
healthy tissues. Univariable and multivariable Cox regression analysis identified several candidate tumor marker genes and prognostic clinical factors. A risk prediction model based on identified genes and clinical factors was constructed. Randomization tests were utilized to calculate P-values of performance metrics, and cross-validation was performed to evaluate the accuracy of the prediction model. The current study integrated genomic and methylation profiles to identify candidate tumor marker genes, which may improve prognosis prediction in patients with CRC.

\section{Materials and methods}

Data sources. Gene expression, DNA methylation profiles and the corresponding clinical information associated with CRC were downloaded from The Cancer Genome Atlas database (TCGA; https://portal.gdc.cancer.gov/). There were 329 samples with both gene expression and DNA methylation profiles, including 41 adjacent healthy tissues and 288 tumor samples. After excluding samples without survival outcome information, the microarray data of the 239 tumor samples were used as the training dataset.

In addition, two validated datasets, GSE77955 (14) and GSE17536 $(15,16)$, were downloaded from Gene Expression Omnibus (https://www.ncbi.nlm.nih.gov/geo/). The GSE77955 dataset included gene expression data based on the GPL96 [hg-u133] Affymetrix Human Genome U133 platform (Affymetrix, Thermo Fisher Scientific, Inc.) and DNA methylation data based on the GPL13534 Illumina Human Methylation 450 BeadChip platform (Illumina, Inc.). A total of 48 pairs of gene expression and methylation profiles were obtained from this dataset, including 14 adjacent healthy tissues and 34 tumor samples. These 48 pairs of samples had matched gene expression levels and DNA methylation levels and corresponding clinical information. This dataset was used as validation dataset of SVM classifier. The GSE17536 dataset consisted of 177 tumor tissue samples with corresponding survival outcomes. The gene expression data were based on GPL570 [HG-U133_Plus_2] Affymetrix Human Genome U133 Plus 2.0 Array. The human gene expression profiles of CRC were selected as validation datasets containing corresponding survival outcome information. This dataset was used as independent validation dataset of prognosis model. Clinical information of samples in the training set and the two validation datasets are presented in Table I.

Differentially expressed mRNAs and methylated genes selection. The edgeR package version 30.2.9 (bioconductor. org/packages/release/bioc/html/edgeR.html) in R software (version no. 3.4.1; https://www.r-project.org/) was used to identify the differentially expressed mRNAs and methylated genes in normal and tumor samples. A false discovery rate (FDR) $<0.05$ and $\mid \log _{2}$ fold change $(\mathrm{FC}) \mid>0.585$ were considered as thresholds. Furthermore, the Wilcoxon signed-rank test (https://www.rdocumentation.org/packages/stats/versions/3.4.1/topics/wilcox.test) in $\mathrm{R}$ was used to identify genes with significant differences in DNA methylation levels between normal and tumor samples. The screening criteria were set as FDR $<0.05$ and ICancer-Normall $>0.2$.
The oligo package version 1.48 (www.bioconductor. org/packages/release/bioc/html/oligo.html) in R was used for preprocessing of the GSE77955 and GSE31056 datasets. Data preparation included original data conversion, missing value supplementation, background correction and data normalization.

Correlation analysis between differentially expressed $m R N A$ and methylated genes. Overlapping differentially expressed mRNA and differentially methylated genes were selected for further analysis. The Cor function (https://www.rdocumentation.org/packages/stats/versions/3.6.1/topics/cor) in $\mathrm{R}$ was used to calculate the Pearson's correlation coefficients (PCC) of gene expression levels and DNA methylated levels. The differentially expressed mRNAs with significantly differential methylated levels were considered as candidate tumor biomarkers.

Construction of a sample classifier based on tumor marker genes. The recursive feature elimination (RFE) algorithm in the caret package version 6.0-84 (17) (cran.r-project. $\mathrm{org} / \mathrm{web} /$ packages/caret) was used to screen tumor marker genes in CRC. The RFE algorithm is an iterative procedure that screens and assesses the optimal subsets from the training dataset (18) and has been employed for dimensionality reduction analysis. The gene combination with the highest accuracy rate in cross validation of RFE algorithm was taken as the optimal combination of tumor markers for validation.

Following the identification of optimal marker genes using RFE algorithm, Support Vector Machine (SVM) package version 1.6.8 (cran.r-project.org/web/packages/e1071) (19) was utilized to construct a SVM classifier (kernel function, sigmoid kernel; cross, 10-fold cross validation) and the performance of SVM classifier was validated using the GSE77955 dataset. Additionally, the pheatmap package version 1.0.8 (bioconductor. org/packages/release/bioc/html/pheatmap.html) (20) in R was used to perform bidirectional hierarchical clustering analysis using the centered Pearson's correlation algorithm (21). In order to investigate the functions of the tumor marker genes, Gene Ontology (GO)-biological processes (BP) and Kyoto Encyclopedia of Genes and Genomes (KEGG) pathways of these genes were analyzed using DAVID version 6.7 (https://david. ncifcrf.gov/) without any cut-off.

Screening tumor marker genes and prognostic clinical factors. To further investigate the optimal tumor marker genes, multivariate and univariate cox regression analysis in the survival package version 2.42-3 (https://cran.r-project. $\mathrm{org} / \mathrm{web} /$ packages/survival/index.html) in $\mathrm{R}$ (22) were performed to assess prognostic values of the selected genes and clinical factors. $\mathrm{P}<0.05$ was considered to indicate a statistically significant difference. Survival curves were generated by the Kaplan-Meier method and log-rank test.

Risk prediction model construction based on tumor marker genes and prognostic clinical factors. The prognostic index (PI) of each sample was calculated according to the following formula: $\mathrm{PI}_{\text {genomic }}=\beta_{\text {gene } 1} \times \operatorname{Exp}_{\text {gene } 1}+\beta_{\text {gene } 2} \times \operatorname{Exp}_{\text {gene } 2}+\beta_{\text {gene } 3} \mathrm{x}$ $\operatorname{Exp}_{\text {gene } 3}+\ldots+\beta_{\text {gene } n} \times \operatorname{Exp}_{\text {gene } n}$, where $\beta$ represents the regression coefficient and Exp represents expression level of a gene. Samples in the training set were divided into high and low risk 
Table I. Clinical characteristics of the training and validation datasets.

\begin{tabular}{|c|c|c|c|}
\hline Clinical characteristic & TCGA $(n=239)$ & GSE77955 $(n=34)$ & $\operatorname{GSE} 17536(n=177)$ \\
\hline Age, years $($ mean $\pm S D)$ & $65.260 \pm 13.170$ & $61.560 \pm 16.050$ & - \\
\hline Sex (male/female) & $130 / 109$ & $18 / 13 / 3$ & - \\
\hline Pathologic M (M0/M1/-) & $159 / 33 / 44 / 3$ & - & - \\
\hline Pathologic N (N0/N1/N2) & $137 / 64 / 38$ & - & - \\
\hline Pathologic T (T1/T2/T3/T4/-) & $5 / 40 / 162 / 31 / 1$ & - & - \\
\hline Pathologic stage (I/II/III/IV/-) & $41 / 88 / 71 / 34 / 5$ & - & - \\
\hline Lymphatic invasion (yes/no/-) & $67 / 151 / 21$ & - & - \\
\hline History of colon polyps (yes/no/-) & $44 / 142 / 53$ & - & - \\
\hline New tumor (yes/no/-) & $51 / 180 / 8$ & - & - \\
\hline Alive (yes/no/-) & $179 / 60 /$ & - & $103 / 74$ \\
\hline Overall survival time (months, mean $\pm \mathrm{SD}$ ) & $29.690 \pm 30.020$ & - & $48.650 \pm 32.460$ \\
\hline
\end{tabular}

TCGA, The Cancer Genome Atlas; SD, standard deviation; -, information unavailable; M, metastasis; N, node; T, tumor.

groups using the median score of the PI as the cut-off value. Kaplan Meier survival curves and long-rank test in the survival package were used to analyze the associations between risk model and disease survival outcome (23). Additionally, receiver operating characteristic (ROC) curves were plotted to calculate the area under the curve (AUC). The GSE17536 dataset was used to perform further data validation.

Based on the risk prediction model of prognostic clinical factors, the PI of each sample was re-calculated according to the following formula: $\mathrm{PI}_{\text {clinical }}=\Sigma \beta_{\text {clinical }}$ x Status clinical $_{\text {, where }} \beta_{\text {clinical }}$ represents the regression coefficient and Status clinical $_{\text {represents }}$ the value of a clinical factor (0 or 1$)$. Samples in the training sets were divided into high- and low-risk groups by using the PI median score as the cut-off value. The Kaplan-Meier survival curves and log-rank test were used to evaluate the associations between the risk model and prognosis. Additionally, ROC curves were plotted to calculate the AUC.

The weight coefficients of optimal tumor marker genes and prognostic clinical factors were integrated and a comprehensive predictive risk model was constructed. The PI value of each sample was re-calculated according to the following formula: $\mathrm{PI}_{\text {combine }}=\mathrm{PI}_{\text {genomic }}+\mathrm{PI}_{\text {clinical }}$. Kaplan-Meier survival curves and log-rank test were generated to evaluate the associations between the risk group and the overall survival (OS) times. ROC curves were plotted to calculate the AUC.

\section{Results}

Screening the differentially expressed mRNAs and differentially methylated genes. A total of 1,706 differentially expressed mRNAs were identified between 329 tumor and adjacent healthy tissues, including 676 upregulated and 1,030 downregulated genes. Moreover, 1,568 differentially methylated genes were screened using the Wilcoxon signed-rank test, including 878 hypomethylated and 690 hypermethylated genes.

Correlation analysis for the gene expression and DNA methylation. There were 228 overlapping differentially expressed mRNAs and differentially methylated genes available for screening the critical tumor marker genes. Correlation analysis was performed to reveal correlations between gene expression and methylation levels (Fig. 1). A total of 54 genes were downregulated with hypermethylated levels, while 164 genes were upregulated with hypomethylated levels. The overall PCC value was -0.668 , suggesting a significant negative correlation between methylation and gene expression levels. The 218 differentially expressed mRNAs with negative correlations between gene expression and methylation levels were considered as candidate tumor biomarkers for further investigation.

SVM method for sample classification. The RFE algorithm was used to identify 47 optimal marker genes from the aforementioned 218 candidate genes. An SVM classifier was constructed using the 47 optimal marker genes, achieving a mean accuracy rate of $100 \%$ (Fig. 2A). ROC curves were plotted to calculate the AUC (Fig. 2B and D). Furthermore, the GSE77955 dataset was used to independently validate the sample classification efficiency, and a mean accuracy of $97.9 \%$ was achieved (Fig. 2C and E).

Cluster analysis was performed based on the expression values and methylation levels of the 47 optimal marker genes. The 239 samples from TCGA were divided into two groups, cluster 1 and cluster 2 (Fig. 3A), including 114 and 125 tumor samples, respectively. Kaplan-Meier survival curves for the samples in the two groups are presented in Fig. 3B. The $\log \mathrm{P}$-value was 0.006, indicating a significant association of the samples with survival outcome. The mean OS time in cluster 1 was significantly longer than that in cluster 2 (33.890 \pm 33.790 months vs. $25.660 \pm 26.640$ months; $\mathrm{P}=0.042$ ). Moreover, the corresponding clinical information of two cohort samples was analyzed. As presented in Table II, the distributions of the node and disease stage were significantly different between two clusters.

GO-BP terms and KEGG analyses were performed for the 47 candidate genes, and a total of 20 biological processes and 4 pathways were identified (Table III; Fig. 3C). The results revealed that these genes were significantly enriched in 'cell adhesion', 'biological adhesion process', 'fatty acid 


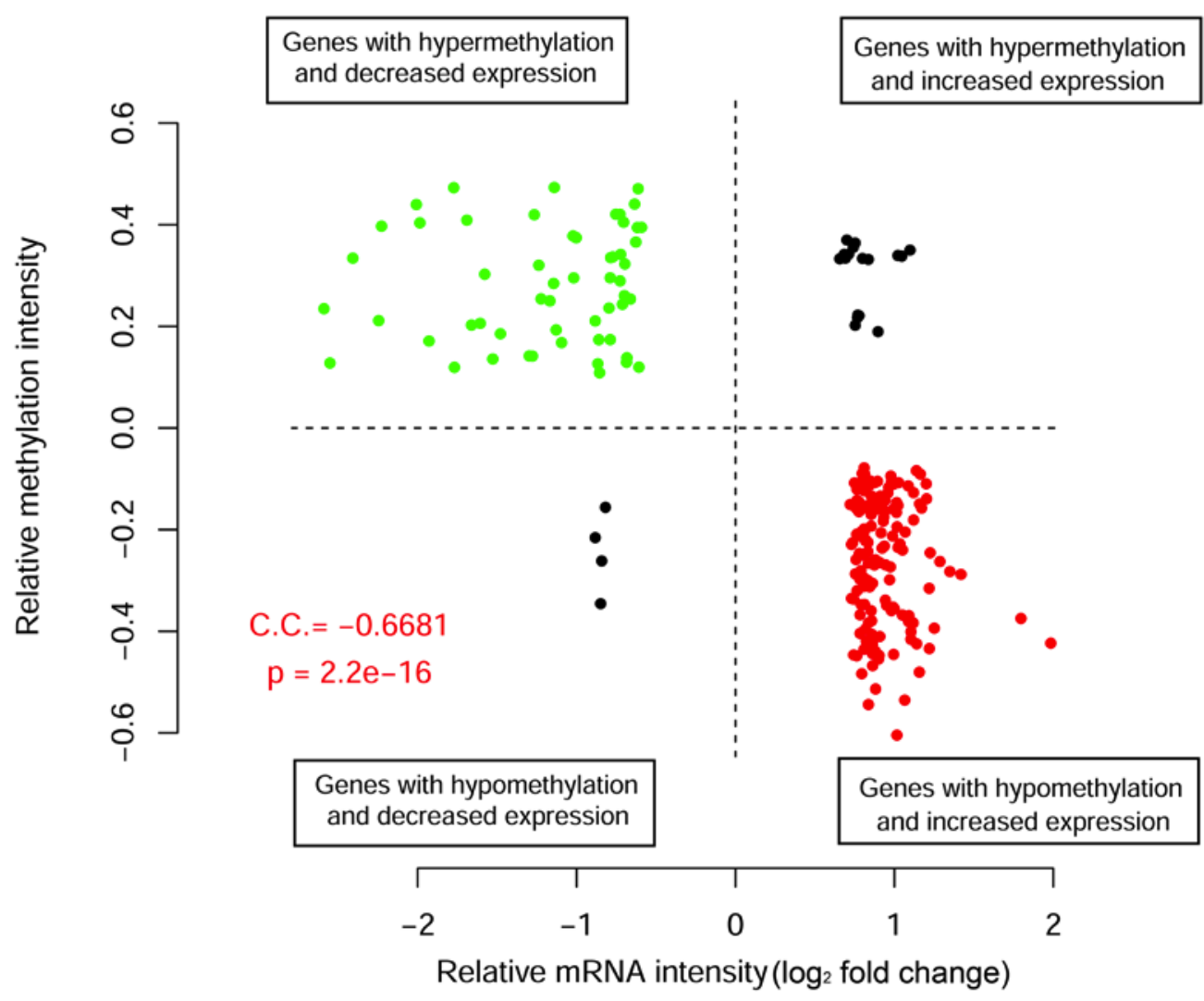

Figure 1. Correlation analysis of gene expression levels with methylation levels for the differentially expressed mRNAs and differentially methylated genes. The horizontal axis represents relative mRNA intensity ( $\log _{2}$ fold change) while the vertical axis represents relative methylation intensity. Green represents hypermethylated genes with decreased expression. Red represents hypomethylated genes with increased expression. Black represents the genes with positive correlations between mRNA levels and methylation levels. CC, correlation coefficient.

metabolism' and 'cytokine receptor interaction signaling' pathways.

Screening prognostic tumor biomarker genes and associated clinical factors. Univariable and multivariable Cox regression analyses were performed to screen tumor biomarker genes and prognostic clinical factors. Eight genes were significantly associated with survival outcomes (Table IV), including endothelial cell-specific molecule 1 (ESM1), pre-B-cell leukemia transcriptional factor 3 (PBX4), acetyl-coenzyme A acyltransferase 2 (ACAA2), chromobox homolog 7 (CBX7), transcriptional enhancer factor TEF-3 (TEAD4), claudin-1 (CLDN1), eukaryotic translated initiation factor 4E family member 3 (EIF4E3) and zymogen granule protein 16 (ZG16). Furthermore, there were six clinical factors associated with OS times according to Kaplan-Meier survival curve analysis, including metastasis stage (M0/M1/-), node stage (N0/N1/N2), tumor stage (T1/T2/T3/T4/-), histological differentiation (I/II/III/IV/-), lymphatic invasion (Yes/No/-) and new tumor (recurrence or not) (Table V; Fig. 4). Furthermore, multivariable Cox regression analysis revealed that new tumor is an independent clinical factor for CRC prognosis $(\mathrm{P}=0.001$; Table $\mathrm{V})$.

Risk prediction model construction based on tumor marker genes. A risk prediction model was constructed based on the eight tumor marker genes identified through Cox regression analysis. The PI of each tumor sample was calculated and the samples in the two training sets were divided into high-risk and low-risk groups with the median score (7.220) of PI as the cut-off value.

Kaplan-Meier survival curves were plotted based on the risk prediction model of prognostic tumor marker genes for the TCGA training set. The results revealed that patients in the low-risk group had a significantly longer OS time compared with those in the high-risk group $(30.060 \pm 30.810$ vs. 21.900 \pm 20.250 months, respectively; $\mathrm{P}=0.009$; Fig. 5A). Prognosis prediction performance of this model was assessed using a ROC curve, and an AUC of 0.981 was obtained (Fig. 5B).

In addition, the OS and disease-free progression survival (DFS) times were validated in the training set GSE17536. For the OS time validation, patients in the low-risk group exhibited a longer OS time compared with patients in the high-risk group (52.510 \pm 31.230 vs. $44.740 \pm 33.380$ months. respectively; $\mathrm{P}=0.025$; Fig. 5C). The ROC curve is presented in Fig. 5C and the AUC was 0.979. Moreover, patients in the low-risk group exhibited a longer DFS time compared with patients in the high-risk group $(42.390 \pm 32.770$ vs. $33.440 \pm 32.590$ months, respectively; $\mathrm{P}=0.008$; Fig. $5 \mathrm{D})$. The AUC value was 0.992 (Fig. 5D).

Risk prediction model construction related to prognostic clinical factors. The risk prediction model of six clinical factors was constructed based on Cox regression. Kaplan-Meier survival curves were used to assess the prognosis prediction performance. Patients in the low-risk group had a longer OS time compared with those in the high-risk group $(30.480 \pm 32.740$ 
A

Recursive feature elimination

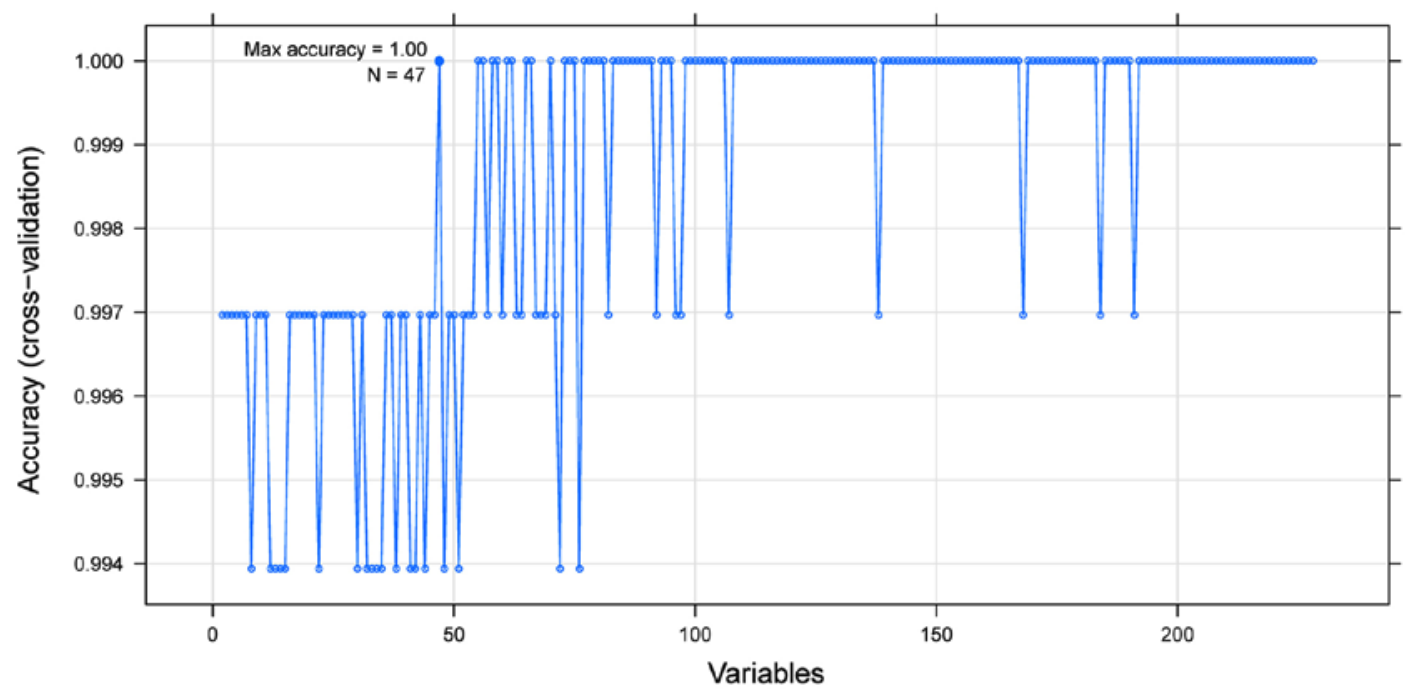

B

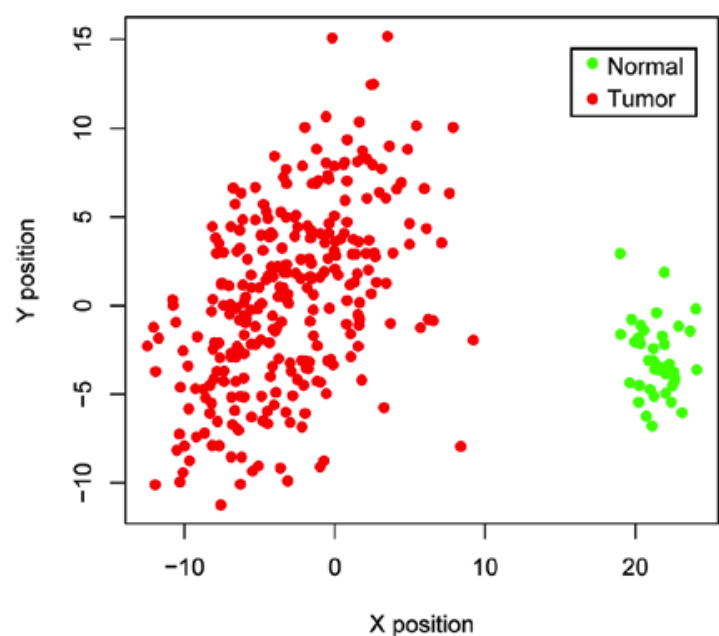

D

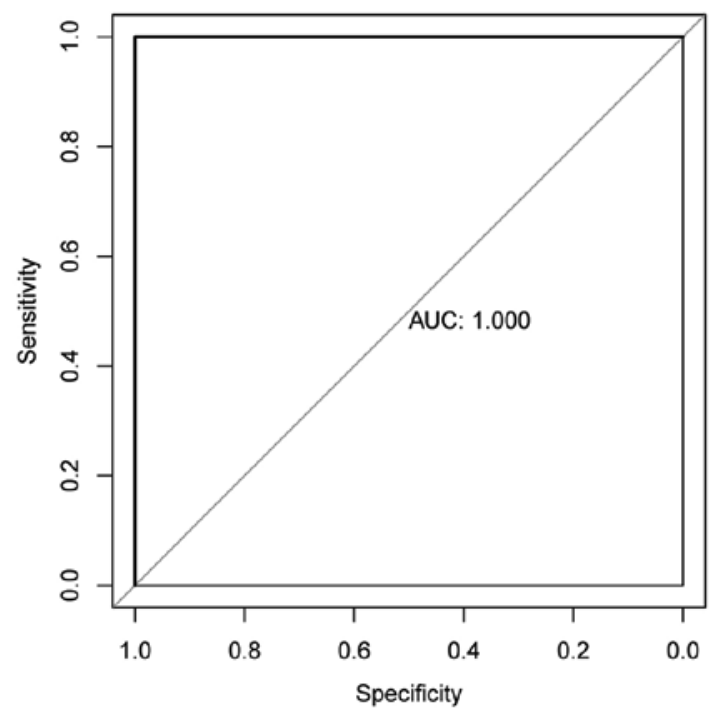

C

Testing set (GSE77953)

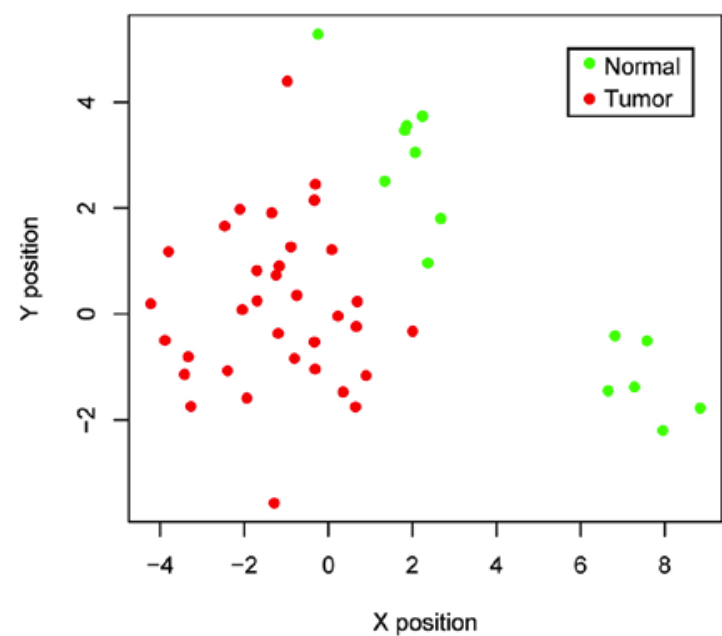

E

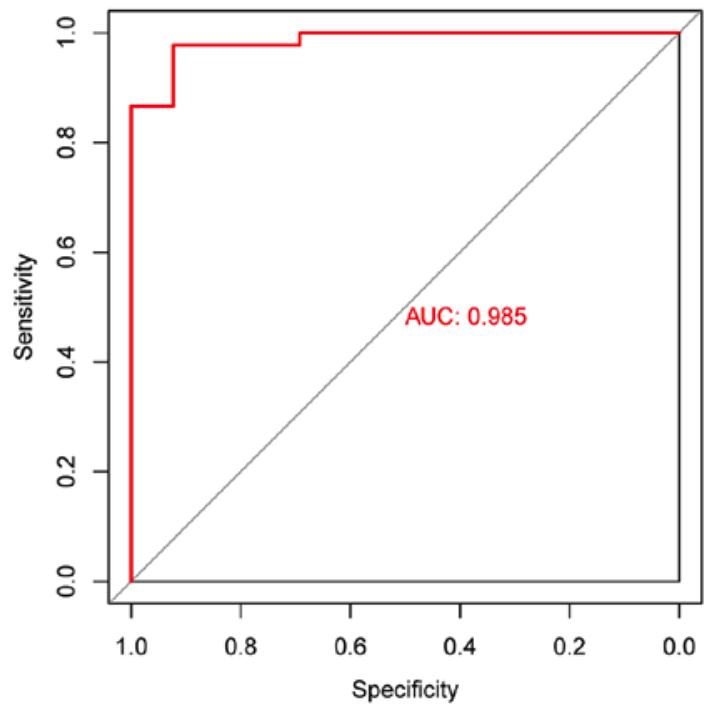

Figure 2. Construction of the SVM classifier for tumor marker genes. (A) Screening the optimal tumor marker genes according to the recursive feature elimination algorithm. (B) Classification scatterplot of samples in the TCGA training set. Red and green dots represent tumor and normal healthy samples, respectively. The $\mathrm{x}$ - and $\mathrm{y}$-axes represent the first dimension and second dimension, respectively. (C) Classification scatterplot of tumor samples in the validation dataset GSE77955. Red and green points represent tumor and normal type samples, respectively. (D) The ROC curve of the SVM classifier for samples in the TCGA training set. (E) The ROC curve of the SVM classifier for samples in the validation set GSE77955. SVM, support vector machine; TCGA, The Cancer Genome Atlas; ROC, receiver operating characteristic. 
A

$\frac{\text { Cluster 1 }}{\text { Cluster 2 }}$

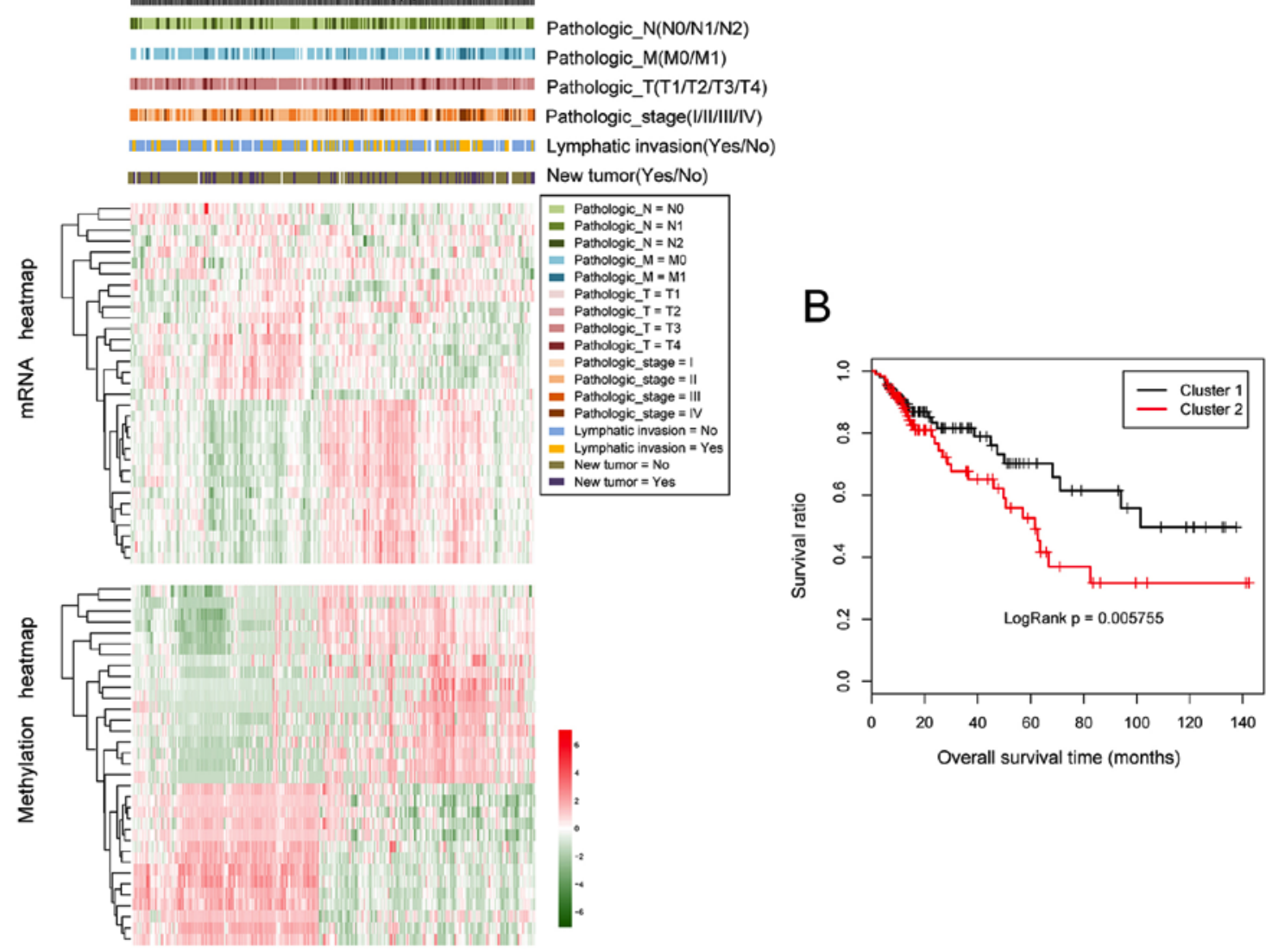

C hsa04670:Leukocyte transendothelial migration
hsa04530:Tight junction hsa04060:Cytokine-cytokine receptor interactionhsa00071:Fatty acid metabolismGO:0051254 positive regulation of RNA metabolic processGO:0051253 negative regulation of RNA metabolic processGO:0051173 positive regulation of nitrogen compound metabolic processGO:0051172 negative regulation of nitrogen compound metabolic process-

GO:0045941 positive regulation of transcriptionGO:0045893 positive regulation of transcription, DNA-dependentGO:0045892 negative regulation of transcription, DNA-dependentGO:0031328 positive regulation of cellular biosynthetic processGO:0031327 negative regulation of cellular biosynthetic processGO:0022610 biological adhesionGO:0016481 negative regulation of transcriptionGO:0010629 negative regulation of gene expressionGO:0010628 positive regulation of gene expressionGO:0010605 negative regulation of macromolecule metabolic processGO:0010604 positive regulation of macromolecule metabolic processGO:0010558 negative regulation of macromolecule biosynthetic processGO:0010557 positive regulation of macromolecule biosynthetic processGO:0009891 positive regulation of biosynthetic processGO:0009890 negative regulation of biosynthetic processGO:0007155 cell adhesion-

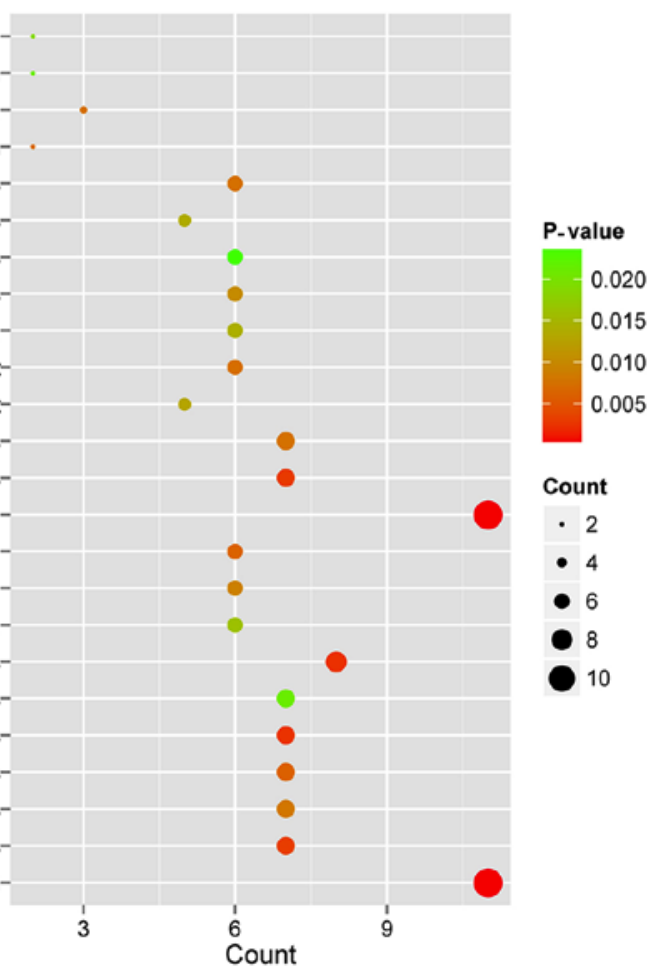

Figure 3. Clustering analysis and enrichment analysis for the tumor marker genes. (A) Bidirectional hierarchical clustering analysis based on the expression values (upper) and methylation levels (lower) of the 47 tumor marker genes. Six clinical factors were successively distributed under the cluster tree, including pathologic N, pathologic M, pathologic T, histological differentiation, lymphatic invasion and new tumor (recurrence or not). Red color represents upregulation and green color represents downregulation. (B) Kaplan-Meier curves presenting the association of tumor samples and survival outcomes in Clusters 1 and 2. (C) GO and Encyclopedia of Genes and Genomes pathway enrichment analysis for the tumor marker genes. The horizontal axis represents the number of genes, while the vertical axis represents the functions and pathways. Dot size represents the number of related genes. Dot color change from red to green represents an increase in the P-value. GO, Gene Ontology. 
Table II. Clinical information and Chi-square test information for cluster 1 and 2 samples.

\begin{tabular}{lrrrrrrrrr}
\hline Clinical characteristic & \multicolumn{3}{c}{ Cluster 1 } & & Cluster 2 & & $\chi^{2}$ & P-value \\
\hline Pathologic M (M0/M1) & 76 & 10 & - & - & 76 & 22 & - & - & 3.018 \\
Pathologic N (N0/N1/N2) & 75 & 18 & 17 & - & 57 & 44 & 20 & - & 13.107 \\
Pathologic T (T1/T2/T3/T4) & 4 & 18 & 78 & 9 & 1 & 19 & 80 & 21 & 6.082 \\
Histological differentiation (I/II/III/IV) & 21 & 48 & 30 & 10 & 18 & 17 & 39 & 23 & 14.306 \\
Lymphatic invasion (yes/no) & 23 & 79 & - & - & 38 & 70 & - & - & 3.474 \\
New tumor (yes/no) & 19 & 88 & - & - & 28 & 88 & - & - & 1.006 \\
\hline
\end{tabular}

M, metastasis; N, node; T, tumor; -, information unavailable.

Table III. GO and KEGG pathways enrichment analysis for 47 candidate tumor genes.

A, GO pathway enrichment analysis

\begin{tabular}{|c|c|c|}
\hline Term & Count & P-value \\
\hline GO: 0007155 cell adhesion & 11 & $<0.001$ \\
\hline GO: 0022610 biological adhesion & 11 & $<0.001$ \\
\hline GO: 0010605 negative regulation of macromolecule metabolic process & 8 & 0.002 \\
\hline GO: 0010558 negative regulation of macromolecule biosynthetic process & 7 & 0.003 \\
\hline GO: 0031327 negative regulation of cellular biosynthetic process & 7 & 0.003 \\
\hline GO: 0009890 negative regulation of biosynthetic process & 7 & 0.003 \\
\hline GO: 0010557 positive regulation of macromolecule biosynthetic process & 7 & 0.006 \\
\hline GO: 0016481 negative regulation of transcription & 6 & 0.006 \\
\hline GO: 0045893 positive regulation of transcription, DNA-dependent & 6 & 0.007 \\
\hline GO: 0051254 positive regulation of RNA metabolic process & 6 & 0.008 \\
\hline GO: $0031328 \sim$ positive regulation of cellular biosynthetic process & 7 & 0.008 \\
\hline GO: 0009891 positive regulation of biosynthetic process & 7 & 0.008 \\
\hline GO: 0010629 negative regulation of gene expression & 6 & 0.009 \\
\hline GO: 0051172 negative regulation of nitrogen compound metabolic process & 6 & 0.010 \\
\hline GO: 0045892 negative regulation of transcription, DNA-dependent & 5 & 0.013 \\
\hline GO: $0051253 \sim$ negative regulation of RNA metabolic process & 5 & 0.014 \\
\hline GO: 0045941 positive regulation of transcription & 6 & 0.014 \\
\hline GO: 0010628 positive regulation of gene expression & 6 & 0.016 \\
\hline GO: 0010604 positive regulation of macromolecule metabolic process & 7 & 0.022 \\
\hline GO: 0051173 positive regulation of nitrogen compound metabolic process & 6 & 0.024 \\
\hline
\end{tabular}

B, KEGG pathway enrichment analysis

\begin{tabular}{lrr}
\hline Term & Count & P-value \\
\hline hsa00071: Fatty acid metabolism & 2 & 0.069 \\
hsa04060: Cytokine-cytokine receptor interaction & 3 & 0.075 \\
hsa04670: Leukocyte transendothelial migration & 2 & 0.191 \\
hsa04530: Tight junction & 2 & 0.214 \\
\hline
\end{tabular}

GO, Gene Ontology; KEGG, Kyoto Encyclopedia of Genes and Genomes.

vs. 24.820 \pm 28.950 months, respectively; $\mathrm{P}<0.001$; Fig. 6A). The ROC curve of prognostic discrimination accuracy of the samples is presented in Fig. 6A, and the AUC was 0.976.
The comprehensive predictive risk model was constructed based on the integrated weight coefficients of the eight tumor marker genes and six clinical factors. The PI of 
Table IV. Candidate tumor marker genes significantly associated with prognosis of colorectal cancer.

\begin{tabular}{|c|c|c|c|}
\hline Gene name & Coefficient & Hazard ratio & P-value \\
\hline Endothelial cell-specific molecule 1 & 0.448 & 1.565 & 0.001 \\
\hline Pre-B-cell leukemia transcriptional factor 3 & 0.341 & 1.406 & 0.007 \\
\hline Acetyl-coenzyme A acyltransferase 2 & -0.413 & 0.662 & 0.008 \\
\hline Chromobox homolog 7 & 0.409 & 1.506 & 0.015 \\
\hline Transcriptional enhancer factor TEF-3 & 0.363 & 1.44 & 0.022 \\
\hline Claudin-1 & 0.124 & 1.132 & 0.038 \\
\hline Eukaryotic translated initiation factor $4 \mathrm{E}$ family member 3 & -0.159 & 0.853 & 0.039 \\
\hline Zymogen granule protein 16 & -0.047 & 0.955 & 0.040 \\
\hline
\end{tabular}

P-value was calculated using log-rank test.
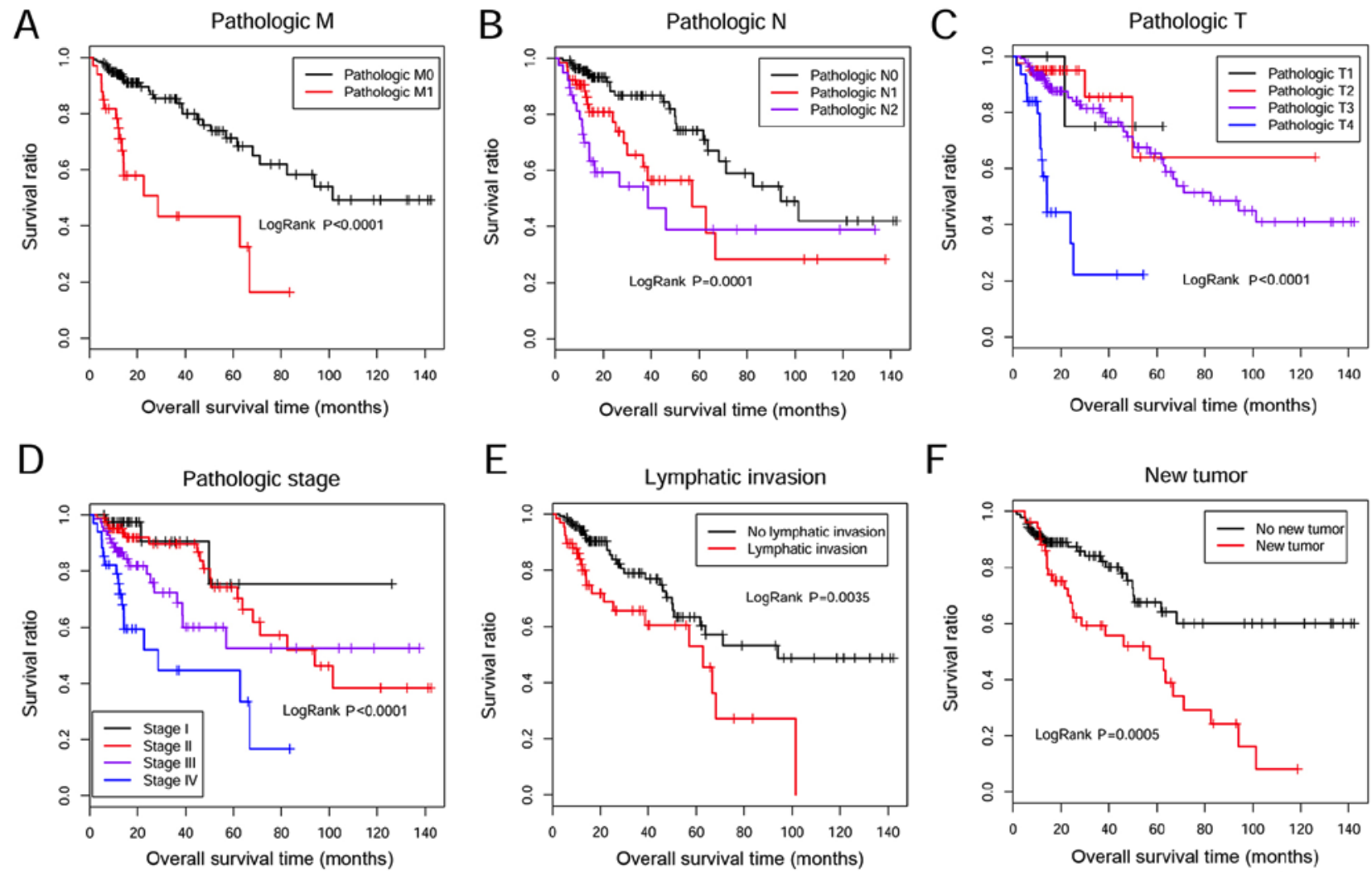

Figure 4. Kaplan-Meier curve analysis for the associations of clinical factors with survival times. The six clinical factors are (A) pathologic M, (B) pathologic $\mathrm{N},(\mathrm{C})$ pathologic $\mathrm{T}$, (D) pathologic stage, (E) lymphatic invasion and (F) new tumor.

each sample was re-calculated and Kaplan-Meier survival curves were plotted to evaluate prognosis prediction performance. Patients in the low-risk group had a longer OS time compared with those in the high-risk group $(31.690 \pm 34.010$ vs. $21.890 \pm 20.710$ months, respectively; $\mathrm{P}<0.001$; Fig. $6 \mathrm{~B})$. The AUC value was 0.999 (Fig. 6B).

\section{Discussion}

In this present study, a total of 218 DEGs with negative correlation between gene expression and methylation levels were identified between CRC and healthy samples. Following the construction of the SVM classifier, 47 genes were further identified as potential tumor marker genes. GO and KEGG enrichment analysis revealed that these genes were mainly associated with 'cell adhesion', 'fatty acid metabolism' and 'cytokine receptor interaction signaling' pathways. Among these genes, univariable and multivariable Cox regression analysis revealed that eight genes (ESM1, PBX4, ACAA2, CBX7, TEAD4, CLDN1, EIF4E3 and ZG16) were significantly associated with the survival times of patients with CRC. The prognostic risk prediction models were constructed based on these genes, six prognostic clinical factors or the combination of the eight marker genes with the six clinical prognostic factors. The performance of each prediction model was evaluated by using the training and validation datasets. The eight 
Table V. Univariable and multivariable Cox regression analysis for prognostic clinical factors.

\begin{tabular}{|c|c|c|c|c|}
\hline \multirow[b]{2}{*}{ Clinical characteristic } & \multicolumn{2}{|c|}{ Univariable cox regression } & \multicolumn{2}{|c|}{ Multivariable cox regression } \\
\hline & P-value & $\mathrm{HR}(95 \% \mathrm{CI})$ & P-value & HR $(95 \% \mathrm{CI})$ \\
\hline Age, years $(\leq 65 />65)$ & 0.137 & $1.501(0.875-2.573)$ & - & - \\
\hline Sex (male/female) & 0.085 & $1.583(0.935-2.683)$ & - & - \\
\hline History of colon polyps (yes/no/-) & 0.312 & $0.616(0.239-1.589)$ & - & - \\
\hline Pathologic M (M0/M1/-) & $<0.001$ & $4.189(2.238-7.839)$ & 0.8242 & $1.143(0.352-3.710)$ \\
\hline Pathologic N (N0/N1/N2) & $<0.001$ & $1.777(1.317-2.399)$ & 0.8034 & $1.149(0.385-3.428)$ \\
\hline Pathologic T (T1/T2/T3/T4/-) & $<0.001$ & $3.375(1.918-5.939)$ & 0.6650 & $1.593(0.194-4.081)$ \\
\hline Histological differentiation (I/II/III/IV/-) & $<0.001$ & $1.919(1.414-2.603)$ & 0.7191 & $1.438(0.199-3.412)$ \\
\hline Lymphatic invasion (yes/no/-) & 0.004 & $2.217(1.282-3.833)$ & 0.8923 & $1.074(0.386-2.991)$ \\
\hline New tumor (yes/no/-) & $<0.001$ & $2.426(1.444-4.076)$ & 0.0011 & $4.599(1.837-11.520)$ \\
\hline
\end{tabular}

-, no information unavailable; HR, hazard ratio; CI, confidence interval; $\mathrm{M}$, metastasis; N, node; T, tumor.

genes were integrated with the six clinical factors, and the accuracy of the integrated risk prediction model increased. The P-value associated with risk grouping and prognosis increased from 0.009 to 0.001 . The AUC value improved from 0.992 to 0.999 , suggesting the comprehensive risk prediction model exhibited a good performance for disease prognosis prediction.

ESM1 is a protein that is mainly expressed on endothelial cells in lung and kidney tissues (24). It serves a major role in endothelium-dependent pathological disorders (25). The prognostic value of serum ESM1 expression in patients with CRC has been previously explored in a Chinese population, and the results revealed that abnormal high expression of ESM1 was significantly associated with histological differentiation, TNM stage, tumor invasion and lymph node metastasis (26). Kang et al (27) demonstrated that activation of the nuclear factor- $\kappa \mathrm{B}$ signaling pathway is regulated by ESM-1, and was associated with cell survival, migration and tumor invasion in CRC. The present study revealed that upregulated ESM1 expression and downregulated methylation levels were observed in CRC tissues compared with adjacent tissues, and the abnormal expression level was associated with the decreased OS time, which was consistent with previous studies $(28,29)$. These results indicated ESM1 expression may aid prognosis prediction for patients with CRC.

The results obtained in the current study revealed that the 47 tumor marker genes are associated with cell adhesion. Claudin proteins are cell membrane proteins associated with tight junctions of epithelial cell polarity $(28,29)$. Reduced cell polarity and cell differentiation are frequently observed in cancer, and are associated with tumor invasion and metastasis (30). Abnormal expression levels of claudin proteins have been reported in several types of adenocarcinomas. For example, upregulation of claudin-3 was reported in ovarian and prostate carcinomas, and downregulated claudin-7 has been found in several types of carcinoma, including thyroid neoplasms, head and neck squamous cell carcinoma and invasive esophageal cancer (31-33). Correlation between CRC and CLDN1 expression has exhibited opposite results.
Shibutani et al (34) demonstrated that low expression of CLDN1 in CRC tissues was correlated with poor prognosis in patients with CRC and Bujko et al (35) revealed that upregulation of CLDN1 was observed in CRC tissues compared with healthy adjacent tissues. Although altered CLDN1 expression has been observed in colon carcinoma, there are few studies investigating the roles of epigenetic changes of this gene in CRC. Hahn-Strömberg et al (36) recently demonstrated that CLDN1 is significantly hypomethylated in CRC tumor samples compared with paired normal mucosa samples. The present study demonstrated that upregulated CLDN1 expression with abnormal low methylation levels in tumor tissue and associated with OS time. Furthermore, CLDN1 hypomethylation was associated with differences in biological properties of the tumor, including pathological stage, metastasis and prognosis.

CBX7 downregulation has been reported in multiple human cancer tissues, including CRC (37). A previous study revealed that the downregulation of $\mathrm{CBX} 7$ was negatively associated with survival outcomes in patients with CRC (38). TEAD4 promotes tumorigenesis by transcriptionally targeting yes-associated protein (YAP)1 in CRC progression (39). Upregulation of TEAD4 may improve its nuclear localization level, subsequently resulting in the epithelial-mesenchymal transition through a YAP-independent manner (40). ZG16 is located on human chromosome 16 and serves as a tumor suppressor by decreasing CRC cell proliferation via its carbohydrate-binding sites (41). Reduced ZG16 expression is associated with pathological phenotypes of CRC (42). Based on the results obtained in the aforementioned studies, CBX7, TEAD4 and ZG16 may be associated with CRC progression. To the best of our knowledge, the current study is the first to systemically demonstrate that the aberrant expression and methylation levels of ESM1, PBX4, ACAA2, CBX7, TEAD4, CLDN1, EIF4E3 and ZG16 are associated with the OS time of patients with CRC. Therefore, these genes may serve as prognostic tumor marker genes.

The current study had a number of limitations. Firstly, the results obtained were not verified by in vitro and in vivo experiments. Secondly, the number of CRC and adjacent normal 

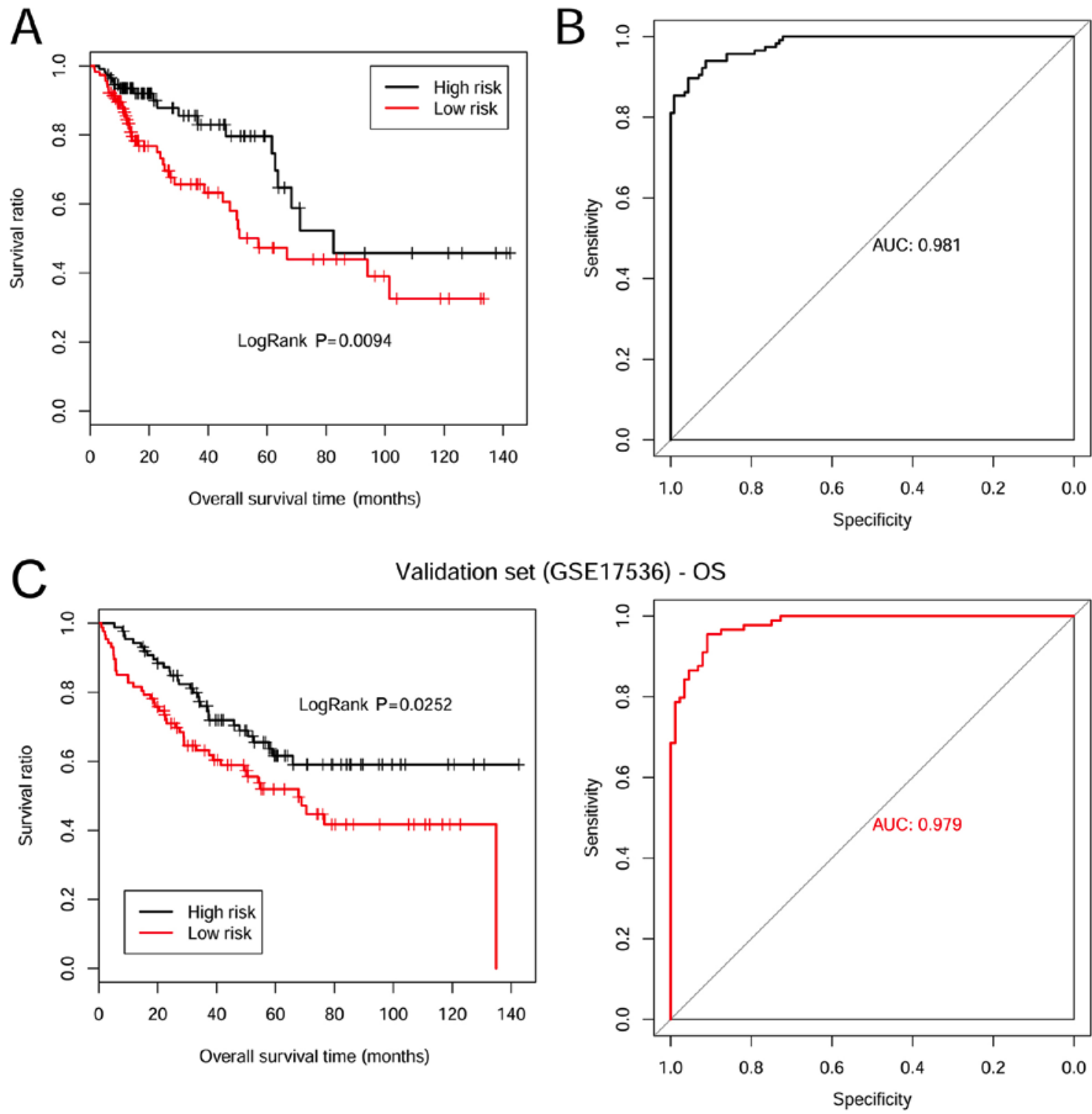

D

Validation set (GSE17536) - DFS
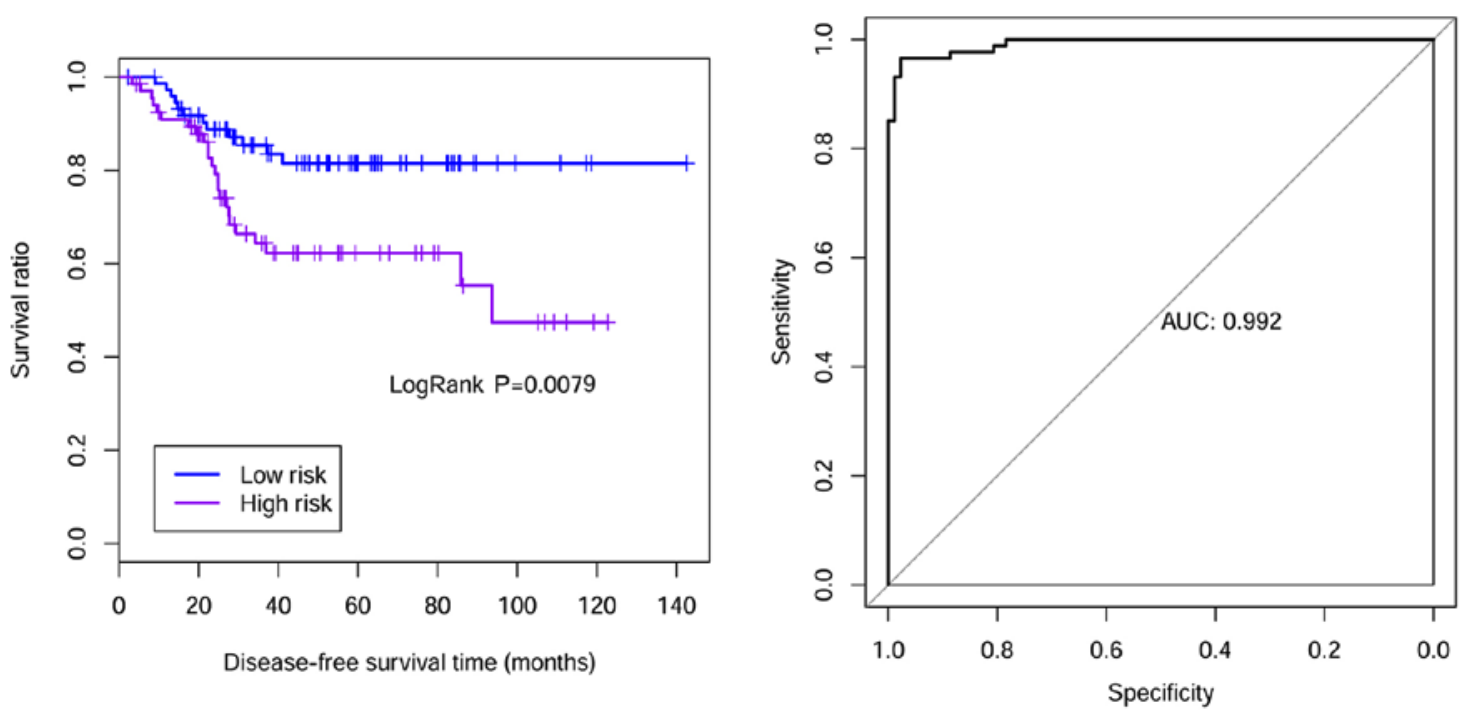

Figure 5. Risk prediction model construction for tumor marker genes and prognostic clinical factors. (A) Kaplan-Meier survival curve analysis for the association of the risk prediction model and overall survival times. (B) ROC curve for the tumor marker genes in the TCGA training set. (C) Kaplan-Meier survival curve (left) and ROC curve (right) for the samples in the validation dataset GSE17536. (D) Kaplan-Meier survival curve (left) and ROC curve for the samples in the GSE17536 dataset. TCGA, The Cancer Genome Atlas; AUC, area under the curve; ROC, receiver operating characteristic; OS, overall survival; DFS, disease-free survival. 

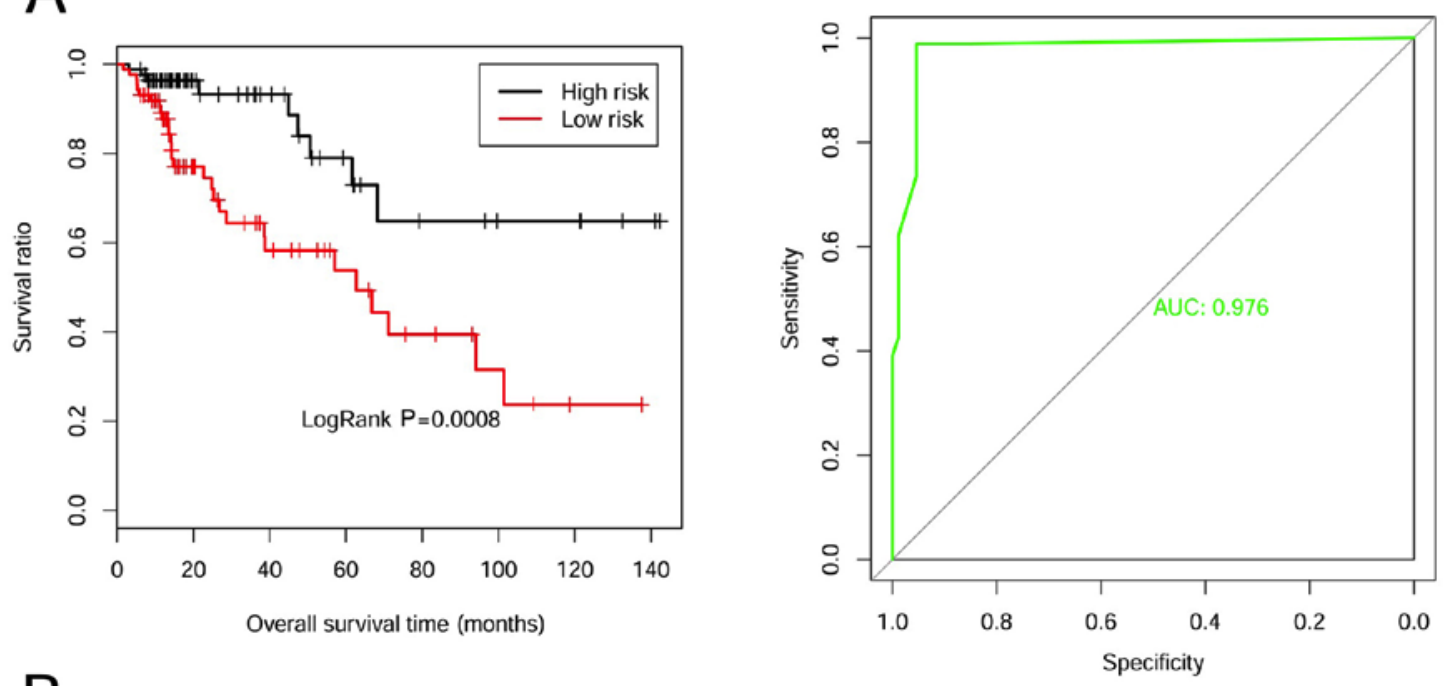

B

Combined genomic model
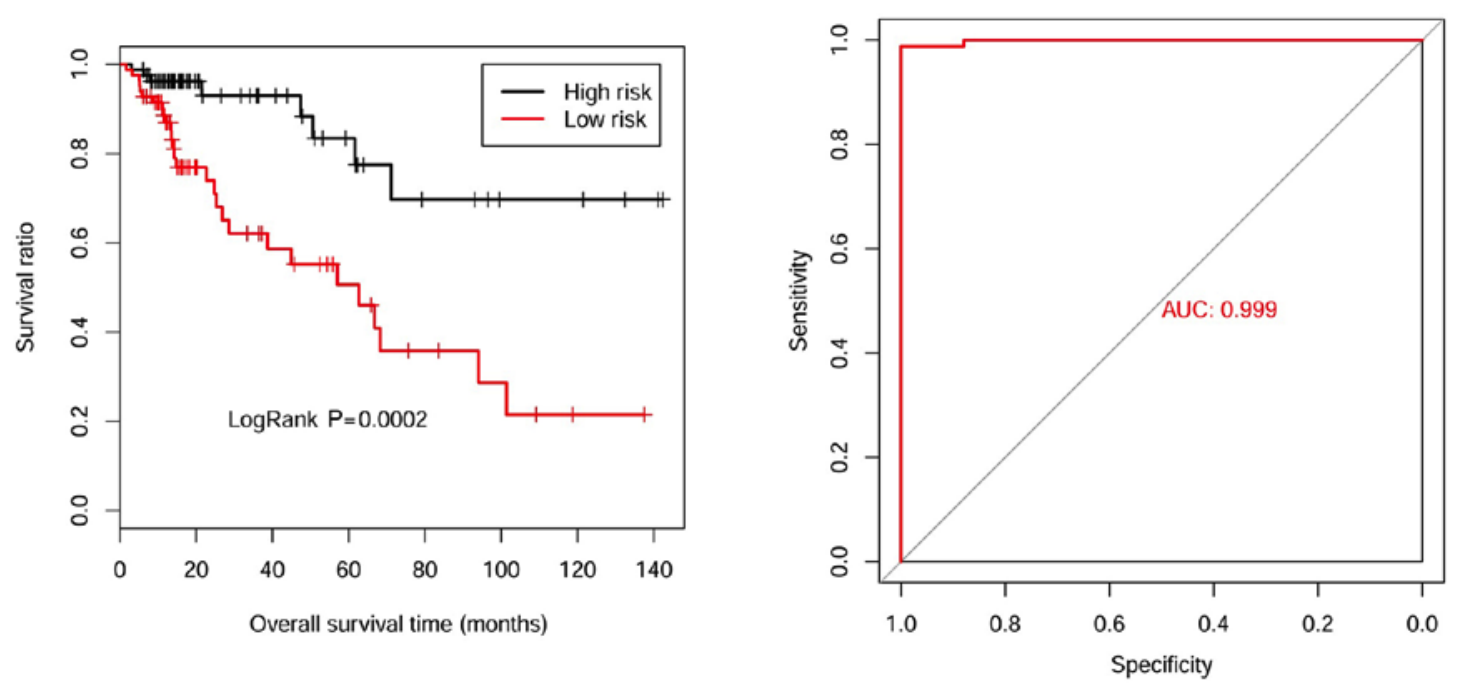

Figure 6. Kaplan-Meier survival curve (left) and ROC curve (right) analysis. (A) Kaplan-Meier survival curve (left) and ROC curve (right) analysis for the association between prognostic clinical factors and overall survival times. (B) Kaplan-Meier survival curve (left) and ROC curve (right) analysis for the association between integrated clinical factors and tumor marker genes. ROC, receiver operating characteristic; AUC, area under the curve.

healthy samples was small. Additionally, since an independent validation dataset containing the six clinical factors is not available, the comprehensive risk prediction model cannot be verified. Future studies integrating more methylation data to study the mechanisms of gene methylation in CRC progression are required.

In summary, the present study identified eight methylated genes (ESM1, PBX4, ACAA2, CBX7, TEAD4, CLDN1, EIF4E3 and ZG16) that may serve as potential tumor marker genes for prognosis prediction of patients with CRC in a clinical setting.

\section{Acknowledgements}

Not applicable.

\section{Funding}

No funding was received.

\section{Availability of data and materials}

The datasets generated or analyzed during the current study are available from the TCGA and GEO repositories (accession nos. GSE GSE77955 and GSE17536).

\section{Authors' contributions}

FX conceived and designed the study. GH and WC performed the data analysis and wrote the manuscript. All authors read and approved the final manuscript.

\section{Ethics approval and consent to participate}

Not applicable.

\section{Patient consent for publication}

Not applicable. 


\section{Competing interests}

The authors declare that they have no competing interests.

\section{References}

1. Arnold M, Sierra MS, Laversanne M, Soerjomataram I, Jemal A and Bray F: Global patterns and trends in colorectal cancer incidence and mortality. Gut 66: 683-691, 2017.

2. Rougier P, Sahmoud T, Nitti D, Curran D, Doci R, De Waele B, Nakajima T, Rauschecker H, Labianca R, Pector JC, et al: Adjuvant portal-vein infusion of fluorouracil and heparin in colorectal cancer: A randomised trial. European Organisation for Research and Treatment of Cancer Gastrointestinal Tract Cancer Cooperative Group, the Gruppo Interdisciplinare Valutazione Interventi in Oncologia, and the Japanese Foundation for Cancer Research. Lancet 351: 1677-1681, 1998.

3. Wood LD, D Williams P, Sian J, Lin J, Sjöblom T, Leary RJ, Shen D, Boca SM, Barber T, Ptak J, et al: The genomic landscapes of human breast and colorectal cancers. Science 318: 1108-1113, 2007.

4. Van Es JH and Clevers H: Notch and Wnt inhibitors as potential new drugs for intestinal neoplastic disease. Trends Mol Med 11: 496-502, 2005

5. Watt FM: Unexpected hedgehog-Wnt interactions in epithelial differentiation. Trends Mol Med 10: 577-580, 2004.

6. Pasparakis M: Role of NF- $\kappa$ B in epithelial biology. Immunol Rev 246: 346-358, 2012.

7. Xie B, Zhao R, Bai B, Wu Y, Xu Y, Lu S, Fang Y, Wang Z, Maswikiti EP, Zhou X, et al: Identification of key tumorigenesisrelated genes and their microRNAs in colon cancer. Oncol Rep 40: 3551-3560, 2018.

8. Moore LD, Thuc L and Guoping F: DNA methylation and its basic function. Neuropsychopharmacology 38: 23-38, 2013.

9. Fellow DHKLDMCR and Genetics SRiC: DNA methylation: A form of epigenetic control of gene expression. Obstetrician \& Gynaecologist 12: 37-42, 2011.

10. Gaudet F, Hodgson JG, Eden A, Jackson-Grusby L, Dausman J, Gray JW, Leonhardt H and Jaenisch R: Induction of tumors in mice by genomic hypomethylation. Science 300: 489-492, 2003.

11. Rodriguez J, Frigola J, Vendrell E, Risques RA, Fraga MF, Morales C, Moreno V, Esteller M, Capellà G, Ribas M and Peinado MA: Chromosomal instability correlates with genome-wide DNA demethylation in human primary colorectal cancers. Cancer Res 66: 8462-9468, 2006.

12. McInnes T, Zou D, Rao DS, Munro FM, Phillips VL, McCall JL, Black MA, Reeve AE and Guilford PJ: Genome-wide methylation analysis identifies a core set of hypermethylated genes in CIMP-H colorectal cancer. BMC Cancer 17: 228, 2017.

13. Xue W, Wu X, Wang F, Han P and Cui B: Genome-wide methylation analysis identifies novel prognostic methylation markers in colon adenocarcinoma. Biomed Pharmacother 108: 288-296, 2018

14. Qu X, Sandmann T, Frierson H Jr, Fu L, Fuentes E, Walter K, Okrah K, Rumpel C, Moskaluk C, Lu S, et al: Integrated genomic analysis of colorectal cancer progression reveals activation of EGFR through demethylation of the EREG promoter. Oncogene 35: 6403-6415, 2016.

15. Smith JJ, Deane NG, Fei WU, Merchant NB, Zhang B, Jiang A, Lu P, Johnson JC, Schmidt C, Bailey CE, et al: Experimentally derived metastasis gene expression profile predicts recurrence and death in patients with colon cancer. Gastroenterology 138: 958-968, 2010.

16. Freeman TJ, Smith JJ, Chen X, Washington MK, Roland JT, Means AL, Eschrich SA, Yeatman TJ, Deane NG and Beauchamp RD: Smad4-mediated signaling inhibits intestinal neoplasia by inhibiting expression of $\beta$-catenin. Gastroenterology 142: 562-571-e562, 2012.

17. Cortés-Ciriano I, Bender A and Malliavin T: Prediction of PARP inhibition with proteochemometric modelling and conformal prediction. Mol Inform 34: 357-366, 2015.

18. Lu X, Yang Y, Wu F, Gao M, Xu Y, Zhang Y, Yao Y, Du X, $\mathrm{Li} \mathrm{C}, \mathrm{Wu} \mathrm{L}$, et al: Discriminative analysis of schizophrenia using support vector machine and recursive feature elimination on structural MRI images. Medicine (Baltimore) 95: e3973, 2016.

19. Wang Q and Liu X: Screening of feature genes in distinguishing different types of breast cancer using support vector machine. Onco Targets Ther 8: 2311-2317, 2015.

20. Wang L, Cao C, Ma Q, Zeng Q, Wang H, Cheng Z, Zhu G, Qi J, $\mathrm{Ma} \mathrm{H}$, Nian $\mathrm{H}$ and Wang Y: RNA-seq analyses of multiple meristems of soybean: Novel and alternative transcripts, evolutionary and functional implications. BMC Plant Biol 14: 169, 2014.
21. Eisen MB, Spellman PT, Brown PO and Botstein D: Cluster analysis and display of genome-wide expression patterns. Proc Natl Acad Sci USA 95: 14863-14868, 1998.

22. Wang P, Wang Y, Hang B, Zou X and Mao JH: A novel gene expression-based prognostic scoring system to predict survival in gastric cancer. Oncotarget 7: 55343-55351, 2016.

23. Goel MK, Khanna P and Kishore J: Understanding survival analysis: Kaplan-Meier estimate. Int J Ayurveda Res 1: 274-278, 2010.

24. Young JN, Young-Ho K, Jin JY, Kang YH, Lee CI, Kim JW, Yeom YI, Chun HK, Choi YH, Kim JH, et al: Identification of endothelial cell-specific molecule-1 as a potential serum marker for colorectal cancer. Cancer Sci 101: 2248-2253, 2010.

25. Lassalle P, Molet S, Janin A, Heyden JV, Tavernier J, Fiers W, Devos R and Tonnel AB: ESM-1 is a novel human endothelial cell-specific molecule expressed in lung and regulated by cytokines. J Biol Chem 271: 20458-20464, 1996.

26. Jiang H, Fu XG and Chen YT: Serum level of endothelial cell-specific molecule-1 and prognosis of colorectal cancer. Genet Mol Res 14: 5519-5526, 2015.

27. Kang YH, Ji Ny, Han SR, Lee CI, Kim JW, Yeom YI, Kim YH, Chun HK, Kim JW, Chung JW, et al: ESM-1 regulates cell growth and metastatic process through activation of NF- $\kappa B$ in colorectal cancer. Cell Signal 24: 1940-1949, 2012.

28. Tsukita S, Furuse M and Itoh M: Multifunctional strands in tight junctions. Nat Rev Mol Cell Biol 2: 285-293, 2001.

29. Feigin ME and Muthuswamy SK: Polarity proteins regulate mammalian cell-cell junctions and cancer pathogenesis. Curr Opin Cell Biol 21: 694-700, 2009.

30. Martin TA and Jiang WG: Tight junctions and their role in cancer metastasis. Histol Histopathol 16: 1183-1195, 2001.

31. Tzelepi VN, Tsamandas AC, Vlotinou HD, Vagianos CE and Scopa CD: Tight junctions in thyroid carcinogenesis: Diverse expression of claudin-1, claudin-4, claudin-7 and occludin in thyroid neoplasms. Mod Pathol 21: 22-30, 2008.

32. Al Moustafa AE, Alaoui-Jamali MA, Batist G, HernandezPerez M, Serruya C, Alpert L, Black MJ, Sladek R and Foulkes WD: Identification of genes associated with head and neck carcinogenesis by cDNA microarray comparison between matched primary normal epithelial and squamous carcinoma cells. Oncogene 21: 2634-2640, 2002.

33. Lioni M, Brafford P, Andl C, Rustgi A, El-Deiry W, Herlyn M and Smalley KS: Dysregulation of claudin-7 leads to loss of E-cadherin expression and the increased invasion of esophageal squamous cell carcinoma cells. Am J Pathol 170: 709-721, 2007.

34. Shibutani M, Noda E, Maeda K, Nagahara H, Ohtani $H$ and Hirakawa K: Low expression of claudin-1 and presence of poorly-differentiated tumor clusters correlate with poor prognosis in colorectal cancer. Anticancer Res 33: 3301-3306, 2013.

35. Bujko M, Kober P, Mikula M, Ligaj M, Ostrowski J and Siedlecki JA: Expression changes of cell-cell adhesion-related genes in colorectal tumors. Oncol Lett 9: 2463-2470, 2015.

36. Hahn-Strömberg V, Askari S, Ahmad A, Befekadu R and Nilsson TK: Expression of claudin 1, claudin 4, and claudin 7 in colorectal cancer and its relation with CLDN DNA methylation patterns. Tumour Biol 39: 1010428317697569, 2017.

37. Guan ZP, Gu LK, Xing BC, Ji JF, Gu J and Deng DJ: Downregulation of chromobox protein homolog 7 expression in multiple human cancer tissues. Zhonghua Yu Fang Yi Xue Za Zhi 45: 597-600, 2011 (In Chinese).

38. Pallante P, Terracciano L, Carafa V, Schneider S, Zlobec I, Lugli A, Bianco M, Ferraro A, Sacchetti S, Troncone G, et al: The loss of the CBX7 gene expression represents an adverse prognostic marker for survival of colon carcinoma patients. Eur J Cancer 46: 2304-2313, 2010.

39. Tang JY, Yu CY, Bao YJ, Chen L, Chen J, Yang SL, Chen HY, Hong J and Fang JY: TEAD4 promotes colorectal tumorigenesis via transcriptionally targeting YAP1. Cell Cycle 17: 102-109, 2018.

40. Liu Y, Wang G, Yang Y, Mei Z, Liang Z, Cui A, Wu T, Liu CY and Cui L: Increased TEAD4 expression and nuclear localization in colorectal cancer promote epithelial mesenchymal transition and metastasis in a YAP-independent manner. Oncogene 35: 2789-2800, 2016.

41. Mito A, Nakano Y, Saitoh T, Gouraud SSS, Yamaguchi Y, Sato T, Sasaki N and Kojima-Aikawa K: Lectin ZG16p inhibits proliferation of human colorectal cancer cells via its carbohydrate-binding sites. Glycobiology 28: 21-31, 2018.

42. Meng H, Li W, Boardman LA and Wang L: Loss of ZG16 is associated with molecular and clinicopathological phenotypes of colorectal cancer. BMC Cancer 18: 433, 2018.

This work is licensed under a Creative Commons Attribution-NonCommercial-NoDerivatives 4.0 International (CC BY-NC-ND 4.0) License. 